OPEN ACCESS

Edited by:

Diogo Monteiro,

Polytechnic Institute of Santarém,

Portugal

Reviewed by: Antonio Hernández-Mendo, University of Malaga, Spain Rafael E. Reigal,

EADE, Spain

${ }^{*}$ Correspondence:

Daniel A. Marinho

marinho.d@gmail.com

Specialty section:

This article was submitted to Movement Science and Sport

Psychology,

a section of the journal

Frontiers in Psychology

Received: 11 May 2020

Accepted: 15 July 2020

Published: 11 August 2020

Citation:

Marinho DA, Barbosa TM, Lopes VP, Forte P, Toubekis AG and Morais JE (2020) The Influence of the

Coaches' Demographics on Young

Swimmers' Performance

and Technical Determinants.

Front. Psychol. 11:1968.

doi: 10.3389/fpsyg.2020.01968

\section{The Influence of the Coaches' Demographics on Young Swimmers' Performance and Technical Determinants}

\author{
Daniel A. Marinho1,2*, Tiago M. Barbosa ${ }^{2,3}$, Vitor P. Lopes ${ }^{2,3}$, Pedro Forte ${ }^{2,3,4}$, \\ Argyris G. Toubekis ${ }^{5}$ and Jorge E. Morais ${ }^{2,3}$
}

\begin{abstract}
${ }^{1}$ Department of Sports Sciences, University of Beira Interior, Covilhã, Portugal, ${ }^{2}$ Research Center in Sports, Health and Human Development (CIDESD), University of Beira Interior, Covilhã, Portugal, ${ }^{3}$ Department of Sports Sciences, Instituto Politécnico de Bragança, Bragança, Portugal, ${ }^{4}$ Department of Sports Sciences, Douro Higher Institute of Educational Sciences, Penafiel, Portugal, ${ }^{5}$ Sports Performance Laboratory, School of Physical Education and Sport Science, National and Kapodistrian University of Athens, Athens, Greece
\end{abstract}

The purpose of this study was to understand the relationship between the coaches' demographics (academic degree and/or coaching level and/or coaching experience) and young swimmers' performance and technical ability. The sample was composed by 151 young swimmers (75 boys and 76 girls: $13.02 \pm 1.19$ years old, $49.97 \pm 8.77 \mathrm{~kg}$ of body mass, $1.60 \pm 0.08 \mathrm{~m}$ of height, $1.66 \pm 0.09 \mathrm{~m}$ of arm span), from seven different clubs. Seven coaches (one per club) were responsible for the training monitoring. Performance and a set of biomechanical variables related to swim technique and efficiency were assessed. The swimmers' performance was enhanced according to the increase in the coaches' academic degree (1: $75.51 \pm 10.02 \mathrm{~s} ; 2: 74.55 \pm 9.56 \mathrm{~s}$; 3: $73.62 \pm 7.64 \mathrm{~s})$, coaching level $(1: 76.79 \pm 11.27 \mathrm{~s} ; 2: 75.06 \pm 9.31 \mathrm{~s} ; 3$ : $73.65 \pm 8.43 \mathrm{~s}$ ), and training experience ( $\leq 5$-y training experience: $75.44 \pm 9.57 \mathrm{~s} ;>5$ y training experience: $74.60 \pm 9.54 \mathrm{~s})$. Hierarchical linear modeling retained all coaches' demographics characteristics as main predictors (being the academic degree the highest: estimate $=-1.51,95 \%$ confidence interval $=-0.94$ to $-2.08, p=0.014$ ). Hence, it seems that an increase in the demographics of the coaches appears to provide them with a training perspective more directed to the efficiency of swimming. This also led to a higher performance enhancement.

Keywords: biomechanics, efficiency, talent identification, youth, swimming

\section{INTRODUCTION}

In youth sports, training and performance are the main topics of interest for researchers and practitioners (Morais et al., 2016). However, less attention is given to the influence that coaches may induce in their athletes. They are the ones bridging researchers and support staff (producing evidence-based knowledge and recommendations) and the athletes (the end users of such knowledge and services) (Crowcroft et al., 2020). 
Coaches are perceived by both athletes and their significant others (i.e., parents) as role models and key players in making sports experience beneficial for children, as well as facilitating the athletes' social and motor development (Fry and Gano-Overway, 2010). Moreover, it was pointed out that a task-orientation environment facilitated by a coach is highly helpful to the commitment toward the sports activity in youth (Martinent and Decret, 2015). However, this begs the question if coaches with a variety of demographics, backgrounds and experiences will be able to empower athletes to engage in effective task-oriented activities and ultimately to achieve success.

Over the long-term development, a mutual dependence is verified based on the athletes' need to acquire knowledge, competence, and experience from the coach, whereas the coaches need to promote performance and success of the athletes based on their own competences and skills (Philippe and Seiler, 2006). A study by Crowcroft et al. (2020) showed that the use of athlete-monitoring tools improved the coaches' prediction to identify performance changes in adult swimmers. However, young athletes do not display a learning or knowledge acquisition pattern like their older counterparts. One can argue that the perception that athletes may have depends on the type of communication used by the coach to convey the message, which can be related to their background (i.e., demographics) (Santi et al., 2014). Indeed, It was argued that an effective coach is seen as being proficient whenever he/she sets targets, or establishes priorities of intervention (Grosso, 2006). Moreover, it was suggested that these professionals need to begin thinking differently not only about how they coach but also about the nature and truth of the knowledge that informs their coaching, including pedagogical and technical content (Denison, 2010; Gilbert and Côté, 2013). Therefore, it can be suggested that coaches with different demographics may denote or use different communication skills and adopt different coaching styles based on the knowledge they acquire over time. To the best of our knowledge, literature does not report yet how the coaches' demographics (e.g., academic training, coaching training, coaching background) can contribute to young swimmers' performance.

Additionally, age-group coaches face the challenge of designing a development program that must feature an effective dose-response. For one side, there is the need to elicit a performance improvement over time (Yustres et al., 2020); on the other, tackling concerns on side effects of heavy and demanding programs, such as musculoskeletal injuries or psychological issues (e.g., burnout) (Monteiro et al., 2018). A well-designed training program should provide appropriate stimulation in order to produce the expected adaptations (Barroso et al., 2015; Whitworth-Turner et al., 2019). In swimming, some concerns have been raised about youth swimming training workload and periodization (Lang and Light, 2010). Literature suggests two training approaches to be employed in age-group swimming: (1) focusing on high training volume (quantity), or alternatively, (2) on training efficiency (quality) (Nugent et al., 2017). On one side of the debate, the argument is that programs based on large mileage would promote sharp improvements in aerobic fitness, but will not make the swimmers faster (Salo and Riewald,
2008). On the other side, literature reports that young swimmers' performance is highly determined by technical factors (i.e., related to swim efficiency) (Morais et al., 2017). Nonetheless, this approach is seen as a long-term development, and as such, it may not yield short-term effects.

A growing body of knowledge has been reporting that the performance enhancement should be focused on the development and consolidation of the technique, based on a long-term development approach (Morais et al., 2017). The determinant factors related to stroke mechanics (i.e., technique) are the best predictors of young swimmers' performance (by 60-85\%) (Morais et al., 2012; Zacca et al., 2020). Therefore, it can be argued that the coaches' demographics might facilitate better performances of swimmers under them, based on the enhancement of efficiency variables rather than large mileages. Nevertheless, a definitive answer to this remains elusive until now. Even though there were some educated guesses by practitioners, we have failed to find evidence-based knowledge that could be aiding an informed decision-making.

Therefore, the main purpose of this study was to understand the relationship between the coaches' demographics (academic degree, coaching level, training experience) in the applied training content and the swimmers' technical ability and performance. It was hypothesized that the existence of significant differences in the coaches' demographics and training experience would have a meaningful contribution to the young swimmers' performance and its determinants; i.e., higher level of education and longer career experience by coaches will be related to better efficiency by the swimmers.

\section{MATERIALS AND METHODS}

\section{Swimmers}

The sample comprised 151 (75 boys and 76 girls) young swimmers $(13.02 \pm 1.19$ years old, $49.97 \pm 8.77 \mathrm{~kg}$ of body mass, $1.60 \pm 0.08 \mathrm{~m}$ of height, $1.66 \pm 0.09 \mathrm{~m}$ of arm span) with $3.36 \pm 0.77$ years of training experience. These were enrolled in a talent identification program, including national record holders, and swimmers participating regularly in regional and national events. Swimmers were assessed at the end of the second macrocycle of a competitive season (winter's peak competition). Parents or guardians and the swimmers themselves signed an informed consent form. All procedures were in accordance to the Declaration of Helsinki regarding human research, and the university ethics board approved the research design.

\section{Coaches}

The coaches' sample was recruited from seven different swim clubs. Coaches did not provide information about the training workload, but all of them followed the same guidelines on young swimmers' training periodization (Chatard and Stewart, 2011). Seven coaches (six males and one female: $31.52 \pm 4.01$ years old, with $7.29 \pm 3.30$ years of training experience), one per swim club, were responsible for designing and monitoring the training program. All of them held a coaching level certification (level 1: two coaches, level 2: three coaches, level 3: two coaches). Four 
held concurrently a bachelor's degree, two a master degree in science, and one a philosophy doctor's degree. The sample was split up according to their academic degree: graduated, 1; master in science, 2; philosophy doctor, 3. Coaching level: level 1, 1; level 2, 2; level 3, 3. Coaching experience: equal to or less than 5 years of coaching experience, $\leq 5$ years; more than 5 years of coaching experience, $>5$ years.

\section{Research Design}

This was a cross-sectional study aiming to understand the relationship between coaches' demographics and swimmers' performance and technical determinants. Besides the $100 \mathrm{~m}$ freestyle event, a comprehensive set of biomechanical variables was selected to be assessed. As aforementioned, such variables account for $60-85 \%$ of young swimmers' performance at frontcrawl stroke, enhancing their importance in youth swimming (Morais et al., 2012; Zacca et al., 2020). Moreover, there is a set of biomechanical variables that are strongly related to swim efficiency and hence deemed as efficiency proxies (Barbosa et al., 2010, 2015): (1) the stroke length (SL) is the distance swam per stroke cycle (Craig and Pendergast, 1979); (2) the stroke index (SI) describes the ability of swimming at a given velocity with the fewest number of strokes possible (Costill et al., 1985); (3) the Froude efficiency $\left(\eta_{\mathrm{F}}\right)$ is the ratio between the useful mechanical work and the total mechanical work; i.e., it is the efficiency at which the thrust is converted into "useful" work (the work to overcome drag force) (Zamparo et al., 2020); and (4) the intracyclic variation of the swim velocity $(d v)$ is the balance of instantaneous thrust (acceleration) and drag (deceleration) (Barbosa et al., 2010). Additionally, young swimmers' technical training is also focused on hydrodynamic resistance, as better performances are related to less water resistance (Morais et al., 2012; Barbosa et al., 2019). Hence, a set of hydrodynamic variables was assessed: (1) the Froude number $\left(F_{\mathrm{r}}\right)$ is a dimensionless variable that is deemed as a wave-making resistance index; (2) the active drag coefficient $\left(C_{\mathrm{Da}}\right)$ quantifies the resistance of a swimmer displacing in water; and (3) the Reynolds number $\left(R_{\mathrm{e}}\right)$ quantifies the water flow status around the swimmer (i.e., the level of turbulence that a swimmer creates displacing through water) (Kjendlie and Stallman, 2008).

\section{Performance}

The $100 \mathrm{~m}$ freestyle event (short course meter, i.e., $25 \mathrm{~m}$ swimming pool) was selected as performance outcome. The time gap between the data collection and the $100 \mathrm{~m}$ freestyle event was no longer than 15 days as reported elsewhere (Morais et al., 2012).

\section{Kinematics and Efficiency}

Swimmers were invited to undergo three maximal trials of $25 \mathrm{~m}$ at front-crawl and push-off start (at least $30 \mathrm{~min}$ of rest). A speedometer string (Swim Speedo-meter; Swimsportec, Hildesheim, Germany) was attached to the swimmers' hip. An in-house built software (LabVIEW, v. 2010) was used to acquire $(f=50 \mathrm{~Hz})$ and display speed-time data over each trial (Barbosa et al., 2016). Data was transferred by a 12-bit resolution acquisition card (USB-6008; National Instruments,
Austin, TX, United States). Afterward, it was imported into a signal processing software (AcqKnowledge v. 3.9.0; Biopac Systems, Santa Barbara, CA, United States). Signal was handled with Butterworth fourth-order low-pass filter (cutoff: $5 \mathrm{~Hz}$ ). Swim velocity $\left(\mathrm{v}\right.$, in $\left.\mathrm{m} \cdot \mathrm{s}^{-1}\right)$ over the trial was measured between the 11th- and 24th-meter mark. The stroke frequency (SF, in $\mathrm{Hz}$ ) was calculated by the number of cycles per unit of time, from the time it takes to complete one full cycle $(f=1 / P$, where $P$ is the period), and afterward converted to $\mathrm{Hz}$. The mean of three consecutive full stroke cycles was afterward used for analysis.

The SL was calculated as SL $=v / \mathrm{SF}$, where SL is the SL $(\mathrm{m})$, $v$ the swim velocity $\left(\mathrm{m} \cdot \mathrm{s}^{-1}\right)$, and SF the stroke frequency $(\mathrm{Hz})$ (Craig and Pendergast, 1979). The $d v$ was computed as follows:

$$
d v=\frac{\sqrt{\frac{\sum_{i}\left(v_{i}-\bar{v}\right) \cdot F_{i}}{n}}}{\frac{\sum_{i} v_{i} \cdot F_{i}}{n}} \cdot 100
$$

where $d v$ is the intracyclic variation of the swim velocity (\%), $v$ is the mean swimming velocity $\left(\mathrm{m} \cdot \mathrm{s}^{-1}\right), v_{\mathrm{i}}$ is the instant swimming velocity $\left(\mathrm{m} \cdot \mathrm{s}^{-1}\right), F_{\mathrm{i}}$ is the acquisition frequency, and $n$ is the number of observations (Barbosa et al., 2010). The SI was computed as follows: $\mathrm{SI}=v \cdot \mathrm{SL}$, where $\mathrm{SI}$ is the $\mathrm{SI}\left(\mathrm{m}^{2} \cdot \mathrm{s}^{-1}\right), v$ the swim velocity $\left(\mathrm{m} \cdot \mathrm{s}^{-1}\right)$, and SL the SL (m) (Costill et al., 1985). The $\eta_{\mathrm{F}}$ was calculated as follows:

$$
\eta_{\mathrm{F}}=\left(\frac{v \cdot 0.9}{2 \pi \cdot S F \cdot l}\right) \cdot \frac{2}{\pi} \cdot 100
$$

where $\eta_{F}$ is the Froude efficiency (\%), $v$ the swim velocity $\left(\mathrm{m} \cdot \mathrm{s}^{-1}\right), \mathrm{SF}$ the stroke frequency $(\mathrm{Hz})$, and $l$ the shoulder to hand average distance (m) (Zamparo et al., 2020). The $l$ was measured between the acromion and tip of the third finger, on dry land, whereas the swimmer was simulating a stroke cycle by digital photogrammetry (Morais et al., 2012).

\section{Hydrodynamics}

The active drag coefficient ( $C_{\mathrm{Da}}$, dimensionless) was computed with the velocity perturbation method (Kolmogorov and Duplishcheva, 1992). Swimmers were invited to perform two maximal trials at front crawl: one trial towing a hydrodynamic body (perturbation device) and the other without (Kolmogorov and Duplishcheva, 1992). The swim velocity was calculated as follows: $v=d / t$. The active $\operatorname{drag}\left(D_{\mathrm{a}}\right.$, in $\left.N\right)$ was computed as follows:

$$
D_{a}=\frac{D_{b} v_{b} v^{2}}{v^{3}-v_{b}^{3}}
$$

where $D_{\mathrm{a}}$ is the swimmers' active drag at maximal velocity $(N) ; D_{\mathrm{b}}$ is the resistance of the hydrodynamic body computed from the manufacturer's calibration of the buoy-drag characteristics and its velocity $(N)$; and $v_{\mathrm{b}}$ and $v$ are the swim velocities with and without the perturbation device $\left(\mathrm{m} \cdot \mathrm{s}^{-1}\right)$. Afterward, the $C_{\mathrm{Da}}$ was computed as follows:

$$
C_{D a}=\frac{2 \cdot D_{a}}{\rho \cdot T T S A \cdot v^{2}}
$$

where $C_{\mathrm{Da}}$ is the active drag coefficient (dimensionless); $D_{\mathrm{a}}$ is the active $\operatorname{drag}(N) ; \rho$ is the density of the water (being $1,000 \mathrm{~kg} \cdot \mathrm{m}^{-3}$ ); 
TTSA is the trunk transverse surface area $\left(\mathrm{m}^{2}\right)$; and $v$ the swim velocity $\left(\mathrm{m} \cdot \mathrm{s}^{-1}\right)$.

The TTSA (in $\mathrm{cm}^{2}$ ) was measured by digital photogrammetry (Morais et al., 2012). The swimmers were invited to put their arms fully extended above the head, one hand over the other; fingers also extended close together and head in neutral position. They were photographed by a digital camera (Alpha 6000; Sony, Tokyo, Japan) in the transverse plane (downward view) on land simulating such streamlined position. Afterward, the TTSA was measured in a specific software (Udruler; AVPSoft, United States) (intraclass correlation coefficient $=0.987$ ).

The Froude number $\left(F_{\mathrm{r}}\right.$, dimensionless) was computed as follows:

$$
F_{r}=\frac{v}{\sqrt{g \cdot H}}
$$

where $F_{\mathrm{r}}$ is the Froude number (dimensionless); $v$ is the swim velocity $\left(\mathrm{m} \cdot \mathrm{s}^{-1}\right), \mathrm{g}$ is the gravitational acceleration $\left(9.81 \mathrm{~m} \cdot \mathrm{s}^{-2}\right)$; and $H$ is the swimmer's height (m) (Kjendlie and Stallman, 2008). The Reynolds number $\left(R_{\mathrm{e}}, \times 10^{6}\right)$ was computed as follows:

$$
R_{e}=\frac{v \cdot H}{v}
$$

where $R_{\mathrm{e}}$ is the Reynolds number (dimensionless); $v$ is the swim velocity $\left(\mathrm{m} \cdot \mathrm{s}^{-1}\right) ; H$ is the height $(\mathrm{m})$; and $v$ is the water kinematic viscosity (being $8.97 \times 10^{-7} \mathrm{~m}^{2} \cdot \mathrm{s}^{-1}$ at $26^{\circ} \mathrm{C}$ ) (Kjendlie and Stallman, 2008).

\section{Statistical Analysis}

The Kolmogorov and Levene tests were applied to check the normality and homoscedasticity assumptions, respectively. Mean +1 standard deviation was computed as descriptive statistics. The relative difference $(\Delta$, in $\%$ ) was calculated to verify the magnitude of the difference between each group of swimmers (i.e., comparison between groups of each coach's demographic characteristics). One-way analysis of variance (ANOVA) $(p<0.05)$ was selected to verify the variation (coach effect) between each coach's demographics (i.e., academic degree and coaching level). Afterward, Bonferroni test $(p<0.05)$ was used to verify differences between pairwise. The independentsample $t$-test $(p<0.05)$ was selected to compare coaching experience (only two groups). The total $\eta^{2}$ was selected as effect size index of the ANOVAs and deemed as follows: (1) without effect if $0<\eta^{2} \leq 0.04$; (2) minimum if $0.04<\eta^{2} \leq 0.25$; (3) moderate if $0.25<\eta^{2} \leq 0.64$; and (4) strong if $\eta^{2}>0.64$ (Ferguson, 2009). Cohen $d$ was selected as a standardized effect size between pairwise comparisons and deemed as (1) small effect size $0 \leq|d| \leq 0.2$, (2) medium effect size if $0.2<|d| \leq 0.5$, and (3) large effect size if $|d|>0.5$ (Cohen, 1988).

The relationship of the coaches' demographics with the performance and technical determinants was computed by hierarchical linear modeling (HLM). This statistical procedure creates a hierarchical structure (i.e., a "tree"), being able to identify the independent variables (coaches' demographics) as performance and technical determinants changing predictors. Two levels were used: (1) the first level included the swimmers' sex; and (2) the second level included the coaches' information (i.e., academic degree, coaching level, and coaching experience). The final model included only significant predictors. Maximum likelihood estimation was calculated with HLM7 software (Raudenbush et al., 2011).

\section{RESULTS}

Table 1 presents the ANOVA (i.e., coach effect) on the coaches' academic degree and coaching level they were holding, as well as the $t$-test comparison between coaching experiences. It also presents the relative difference between groups, on each coach's demographics.

Figure 1 depicts the variables assessed, clustered by the academic degree coaches were holding. The swimmers' performance had an improvement (i.e., swam faster) with an increase in the coaches' academic background (1: $75.51 \pm 10.02 \mathrm{~s}$; 2: $74.55 \pm 9.56 \mathrm{~s} ; 3: 73.62 \pm 7.64 \mathrm{~s}$ ), but without a significant variation (Table 1 ). The $C_{\mathrm{Da}}$ presented a significant variation, with significant differences between groups 1 and $2(p=0.015$, $\Delta=31.58 \%, d=0.69)$, and between groups 1 and $3(p=0.017$, $\Delta=38.89 \%, d=0.84$ ) (Table 1).

Figure 2 depicts the selected variables, clustered by the coaching level held. The swimmers' performance also improved based on the coaching level (1: $76.79 \pm 11.27 \mathrm{~s} ; 2: 75.06 \pm 9.31 \mathrm{~s}$; 3: $73.65 \pm 8.43 \mathrm{~s})$. The $C_{\mathrm{Da}}$ again denoted a significant variation, where significant differences were verified between groups 1 and $2(p=0.012, \Delta=28.30 \%, d=0.92)$, and between groups 2 and 3 $(p<0.001, \Delta=43.24 \%, d=0.93)$ (Table 1).

Figure 3 presents the selected variables, clustered by coaching experience. Swimmers' performance was better under a coach with longer careers ( $\leq 5$ training experience: $75.44 \pm 9.57 \mathrm{~s}$ vs. $>5$ training experience: $74.60 \pm 9.54 \mathrm{~s} ; 1.13 \%)$. The $d v$ showed a significant difference between groups $(t=2.73, p=0.007$, $\Delta=14.88 \%, d=0.45$ ) (Table 1).

In summary, based on bivariate analyses, swimmers benefited from being under coaches holding higher academic degrees, higher coaching levels, and longer careers. The follow-up question was how these factors could interact. To address such question, multivariate analysis (such as HLM modeling) was run.

Table 2 shows the data retrieved from the HLM model about the relationship between the coaches' demographics and young swimmers' performance. The model retained as main predictors all the coaches' demographics [academic degree: estimate $=-1.51$, $95 \%$ confidence interval $(\mathrm{CI})=-0.94$ to $-2.08, p=0.014$; coaching level: estimate $=-0.92,95 \% \mathrm{CI}=-0.45$ to $-1.39, p=0.031$; coaching experience: estimate $=-0.28,95 \% \mathrm{CI}=-0.14$ to -0.42 , $p=0.027$ ] (Table 2). Moreover, the coaching experience also had a significant contribution to the swimmers' $d v$ (estimate $=-0.12$, $95 \% \mathrm{CI}=-0.15$ to $-0.04, p=0.029)$.

\section{DISCUSSION}

The main purpose of this study was to understand the relationship between the coaches' demographics and swimmers' 


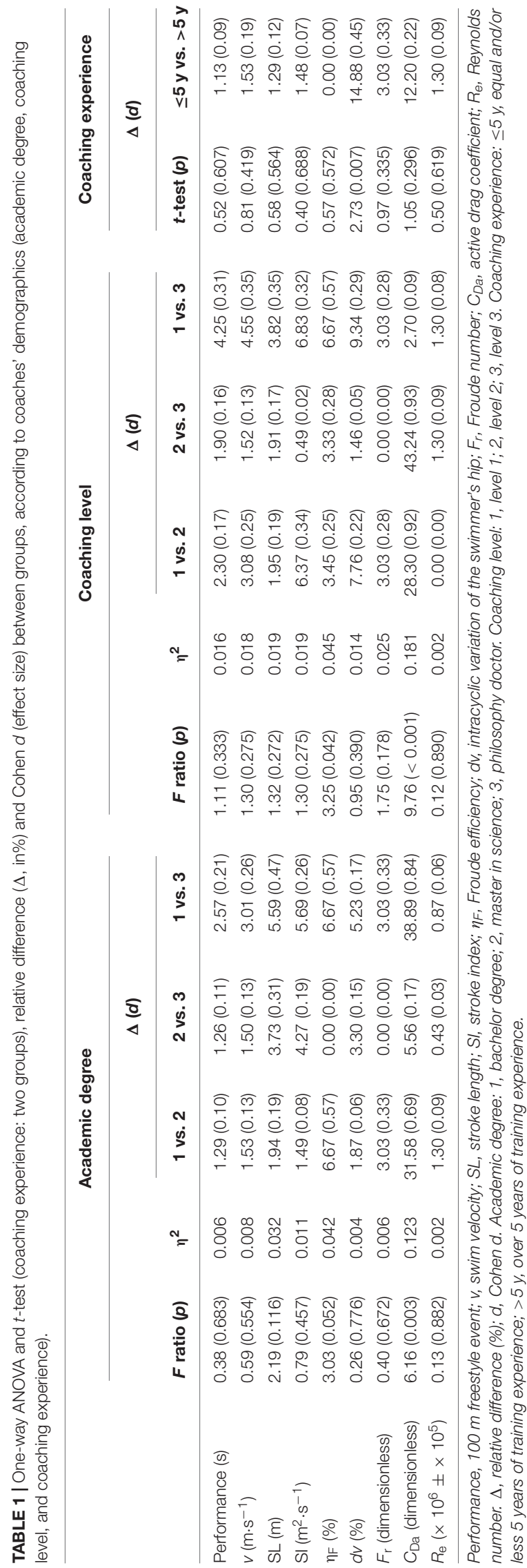

performance, swimming efficiency, and hydrodynamics. HLM suggested that an increase in all the coaches' demographics had a positive and significant effect on young swimmers performance (being the academic degree the highest contributor). This contribution to the performance improvement is related to the enhancement of the variables related to swimming efficiency and hydrodynamics.

Our data showed that when swimmers were grouped by coaches' demographics, an increase in each characteristic was related to an enhancement of the performance and all selected efficiency and hydrodynamics outcomes (Figures 13). Swimmers showing the best performances and better swimming efficiency and hydrodynamics were trained by the most experienced and qualified coaches. This suggests that these coaches may have a training perspective based on the efficiency and hydrodynamic enhancement, that is, quality of the swim technique, rather than in high volumes (quantity), leading to better performances (Nugent et al., 2017). Indeed, literature suggests that a long-term development approach, underpinned by high levels of technical efficiency and hydrodynamics, should be the bedrock of youth sports (Lang and Light, 2010; Morais et al., 2017).

Previous to the 2012 Youth Olympic Games, the excessive training loads in detriment of the development of technique got some attention based on the amount of mileage that young swimmers were being submitted to (Lang and Light, 2010). It was pointed out that 11 - and 12 -years-old swimmers may swim up to 32 miles a week $(51.50 \mathrm{~km})$, and 14 years about 40 miles a week $(64.37 \mathrm{~km})$ (Cassidy, 2008). This large mileage raises two major concerns: (1) it is not in tandem to what is suggested by literature, and (2) it could lead to a high ratio of dropouts. A study reported that 11- to 12years-old swimmers should swim about $25 \mathrm{~km}$ per week (15.53 miles), and 13- to 14 -years-old about $30 \mathrm{~km}$ (18.64 miles) (Chatard and Stewart, 2011). Thus, young swimmers were training $48.54 \%$ ( $11-12$ years) and $46.60 \%$ (13-14 years) more than literature guidelines.

A study showed that during a 3-year period, time (i.e., training) did not have a significant effect on young swimmers' performance enhancement (Morais et al., 2017). The variables that explained and predicted the performance were anthropometrics and stroke mechanics. The authors argued that growth and maturation and its interaction with biomechanics are the major drivers to performance enhancement. While growing, young swimmers will need to "relearn" and readapt their biomechanics to accommodate the shifts in anthropometric features. Coaches play a major role in such process (Morais et al., 2017). Moreover, the excessive amount of mileage can lead to a dropout phenomenon, because it removes the attraction of the sport, leading to a physical and mental burnout. Indeed, swimmers cite the emphasis on frequent, intense training as a main reason for dropping out (Cassidy, 2008). A study conducted with young swimmers noted that "demands," "pressure," and "dissatisfaction" were the dimensions or issues that better characterized the dropout and negatively predicted the intention of return (Monteiro et al., 2018). Moreover, such amount of training at early ages 

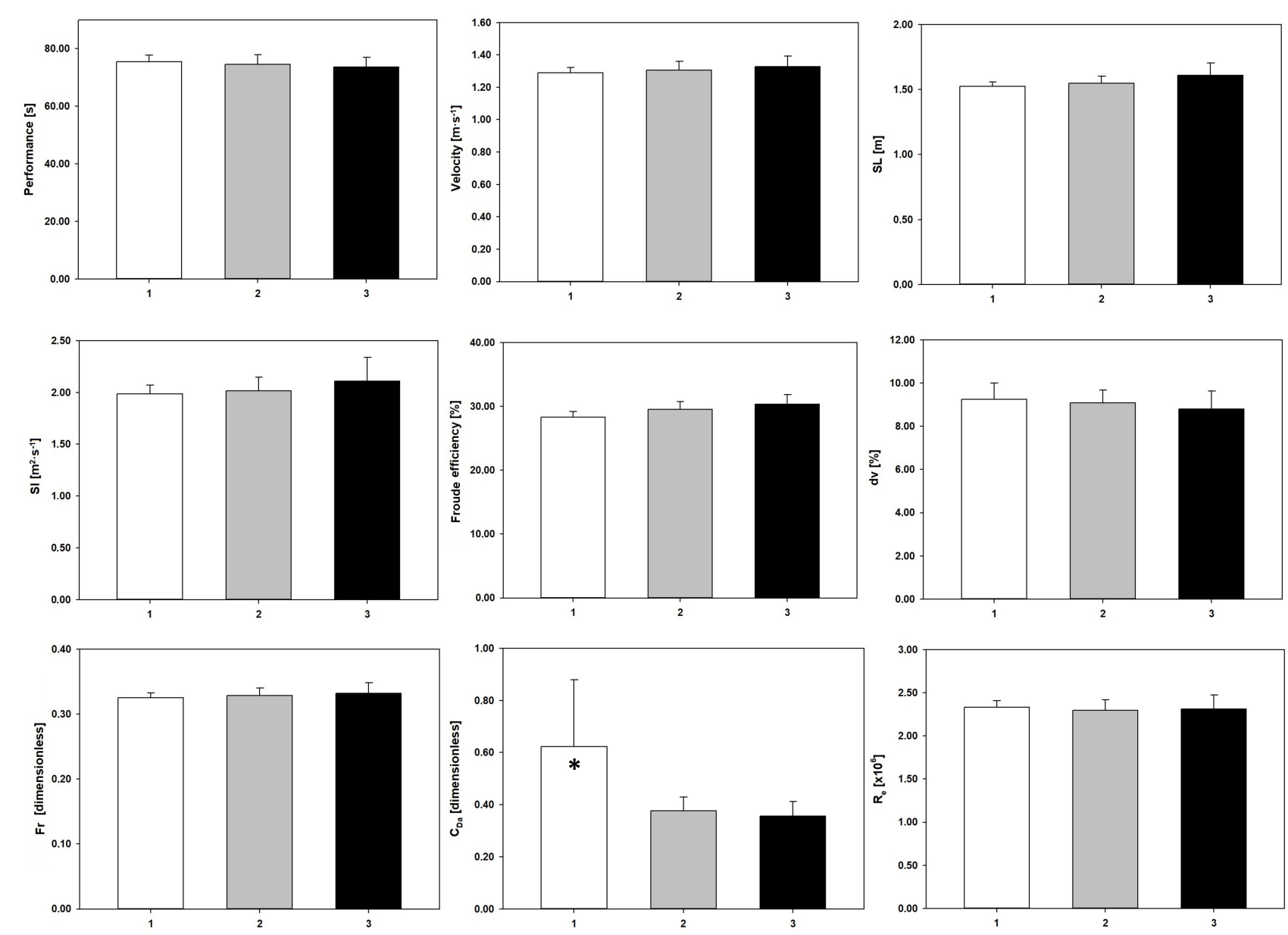

FIGURE 1 | Performance and efficiency variables clustered by the coaches' academic degree. Performance, 100 m freestyle performance; velocity, swim velocity; $\mathrm{SL}$, stroke length; SI, stroke index; $\eta_{\mathrm{F}}$, Froude efficiency; $d v$, intracyclic variation of the swimmer's velocity; $F_{\mathrm{r}}$, Froude number; $C_{\mathrm{Da}}$, active drag coefficient; $R_{\mathrm{e}}$, Reynolds number. 1, bachelor degree; 2, master in science; 3, philosophy doctor. *Significant differences $(p<0.05)$ between: 1 vs. 2 , and 1 vs. 3.

may inhibit musculoskeletal development and increase the likelihood of injuries. This will negatively impact performance and may present negative consequences in the swimmer's growth development (Valovich et al., 2011).

All the variables related to the coach entered the final HLM as main predictors (Table 2). From those, the coaches' academic degree had the highest contribution. An increase in the academic degree was related to a 1.51-s performance enhancement. These data showed that further academic studies led to an enhancement of the swimmers' performance. It could be argued that the amount of knowledge about the performance determinant factors and a higher awareness on how to apply this knowledge and assessment procedures, that is, translating evidence into practice, facilitated swimmers' improvement (Crowcroft et al., 2020). Additionally, coaching courses also allow coaches to gather substantial information not only on training volumes and intensity, but also on technical training (especially regarding young swimmers based on a long-term development). The manipulation of swimmers' training volume (including aerobic and technical training) should promote physical adaptions through progressive overload (Carter et al., 2014). However, and at the same time, a key factor to a successful long-term development requires coaches to use relevant training load and specific training based on technical task-oriented drills (Chatard and Stewart, 2011; Lloyd et al., 2016). Thus, coaches who maintain an updated state-of-the-art about swimming training and long-term development may accurately prescribe and evaluate session's intensity and orientation to avoid an inappropriate approach.

On the other hand, it was suggested that the barriers coaches face to access to sport science (for those who do not hold high academic degree, for instance) are the time required to find and read scientific journals and the lack of direct access to highly trained support staff, such as sport analysts, such as biomechanists (Reade et al., 2008). In the case of competitive swimming, young swimmers' performance is highly driven by anthropometrics and biomechanics (variables related to technique, such as kinematics, hydrodynamics, and efficiency) (e.g., Morais et al., 2012; Barbosa et al., 2019). Thus, it seems that coaches who are aware and familiar with cutting-edge evidence 

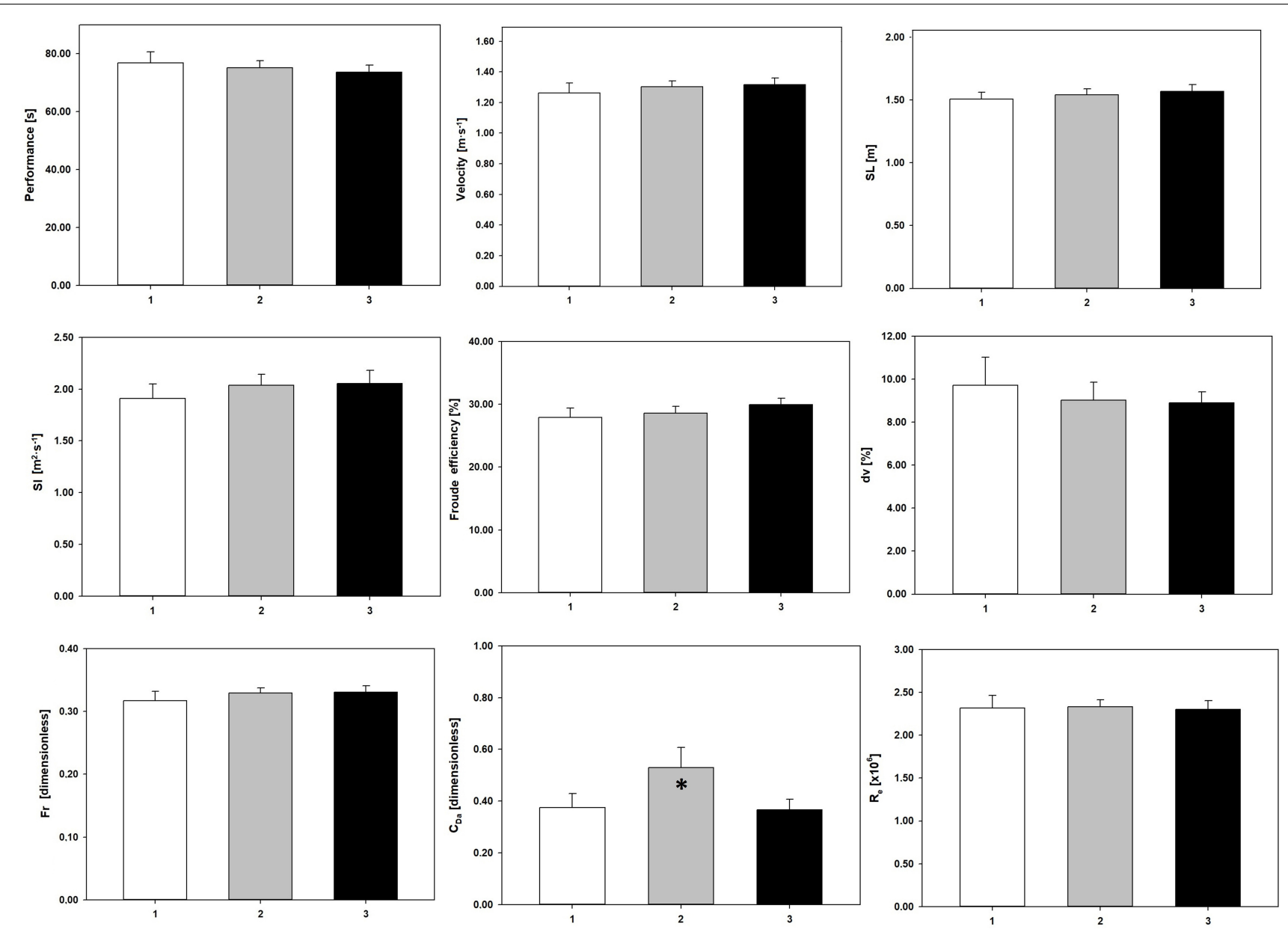

FIGURE 2 | Performance and efficiency variables clustered by the coaches' coaching level. Performance, $100 \mathrm{~m}$ freestyle performance; velocity, swim velocity; SL, stroke length; SI, stroke index; $\eta_{\mathrm{F}}$, Froude efficiency; $d v$, intracyclic variation of the swimmer's velocity; $F_{r}$, Froude number; $C_{\mathrm{Da}}$, active drag coefficient; $R_{\mathrm{e}}$, Reynolds number. 1, coaching level 1; 2, coaching level 2; 3, coaching level 3. *Significant differences $(p<0.05)$ between: 2 vs. 1 , and 2 vs. 3.

about swimming determinants and have the skills to translate it into practice may have an edge in comparison to their peers.

The coaching experience showed a significant and inverse contribution to the swimmers' $d v$ (estimate $=-0.12, p=0.029$ ) (Table 2). The $d v$ is considered as an efficiency proxy (Barbosa et al., 2010). A larger intracyclic variation is related to more energy cost of transportation. So, it seems that coaching experience also provides coaches with knowledge to understand in which way they can help out their swimmers to improve the stroke mechanics, with the goal to minimize $d v$ and therefore to excel.

Overall, it can be pointed out that despite all coaching features entered as significant predictors of young swimmers' performance, the academic degree (i.e., level of scientific knowledge) was the highest contributor. Literature reported that young swimmers' performance is based on interactions of several determinants and is deemed as a dynamic and highly complex system (Barbosa et al., 2010; Morais et al., 2017). Hence, a coach should be able to provide their athletes with training on key skills and abilities based in such determinant factors
(Rezania and Gurney, 2014). In the past decade, research on age-group swimmers has been strongly focused on identifying and modeling the performance determinants (Morais et al., 2017; Barbosa et al., 2019) and analyzing young swimmers' performance based on a talent identification approach (Yustres et al., 2020). However, little attention was given until now to coaches. They should facilitate the development of swimmers under them, designing programs that are underpinned by highlevel and cutting-edge evidence. Coaches should be familiar with the most recent state of-the-art to help their swimmers to excel. Therefore, governing bodies such as national federations and regional associations should play an important role on (1) advising coaches to attend high-level coaching courses or preferably enrolling in higher academic degrees; (2) holding or sponsoring hands-on workshops based on cutting-edge evidence and who translate it to practice; and (3) supporting coaches by facilitating access to new trends and novel and impactful knowledge in youth swimming research.

As main limitations, the lack of information on training programs designed by the recruited coaches (e.g., the disclosure 

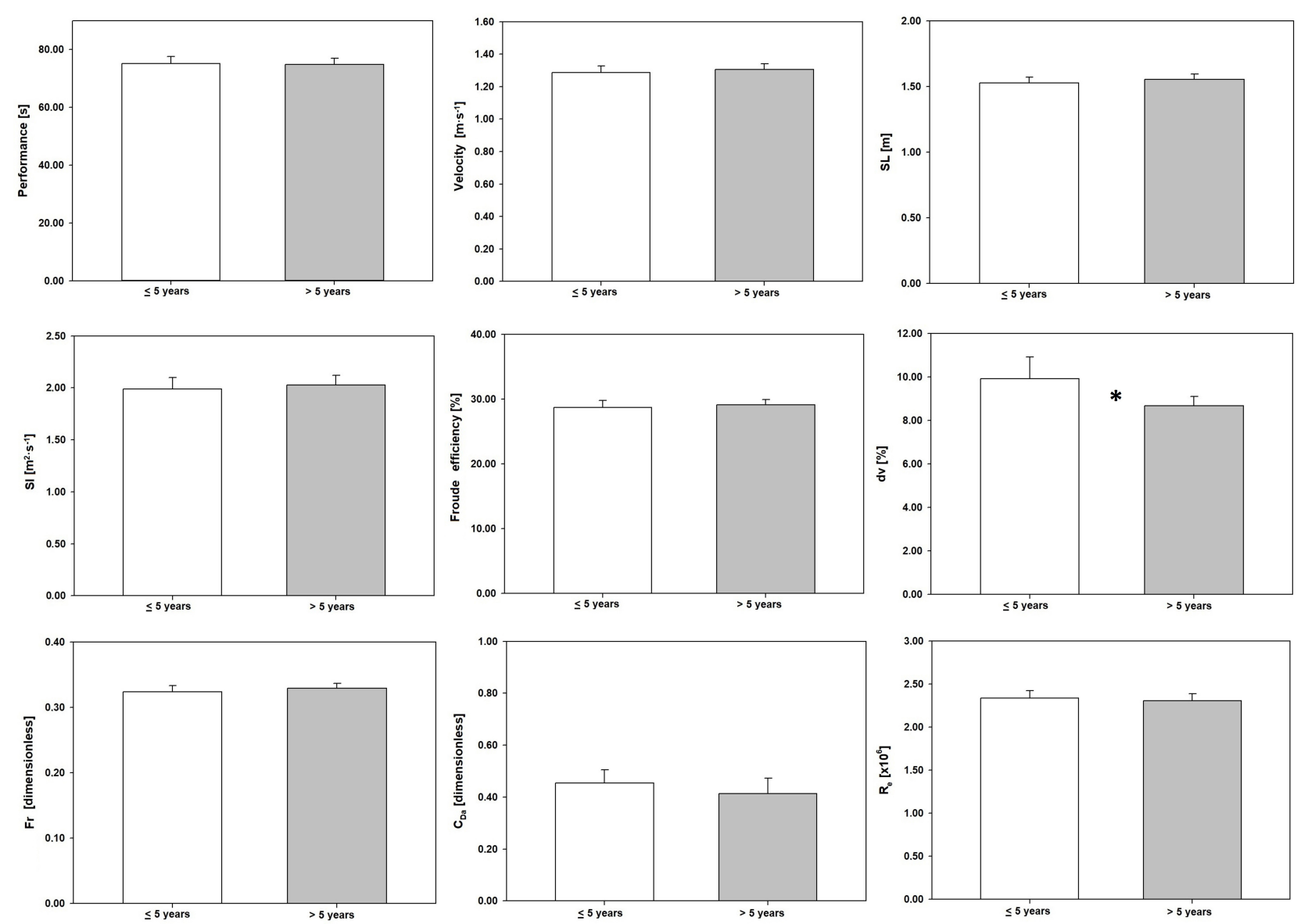

FIGURE 3 | Performance and efficiency variables clustered by the coaches' training experience. Performance, 100 m freestyle performance; velocity, swim velocity; $\mathrm{SL}$, stroke length; SI, stroke index; $\eta_{\mathrm{F}}$, Froude efficiency; $d v$, intracyclic variation of the swimmer's velocity; $F_{r}$, Froude number; $C_{\mathrm{Da}}$, active drag coefficient; $\mathrm{R}_{\mathrm{e}}$, Reynolds number. $\leq 5$ years, 5 or less years of training experience; $>5$, more than 5 years of training experience. $*$ Significant differences $(p<0.05)$.

of the mileage covered by swimmers) and that these estimations are only for the $100 \mathrm{~m}$ freestyle event can be considered. The following can be suggested: (1) a cluster analysis in the future to understand if the fastest swimmers were characterized by higher technical/efficiency parameters and under the coaches with the highest expertise and experience and (2) the assessment

TABLE 2 | Fixed effects of the final models computed with standard errors (SE) and $95 \%$ confidence intervals $(95 \mathrm{Cl})$.

\begin{tabular}{lccc}
\hline Fixed effect & Estimate (SE) & 95CI & $\boldsymbol{p}$-value \\
\hline Performance model & & & \\
Intercept & $79.46(0.81)$ & $77.87-81.05$ & $<0.001$ \\
Academic degree & $-1.51(0.29)$ & -0.94 to -2.08 & 0.014 \\
Coaching level & $-0.92(0.24)$ & -0.45 to -1.39 & 0.031 \\
Coaching experience & $-0.28(0.07)$ & -0.14 to -0.42 & 0.027 \\
$\boldsymbol{d} \boldsymbol{v}$ model & & & \\
Intercept & $9.27(0.12)$ & $(9.03-9.51)$ & $<0.001$ \\
Coaching experience & $-0.12(0.03)$ & $(-0.15$ to -0.04$)$ & 0.029 \\
\hline
\end{tabular}

$d v$, intracyclic variation of the swim velocity. of psychological variables to learn the relationship between the coaches' demographics (academic degree, coaching level, training experience) in the applied training content and the swimmers' psychological profile and performance.

\section{CONCLUSION}

The coaches' demographics (i.e., academic degree, coaching level, and coaching experience) have a significant and positive relationship with young swimmers' performance and swimming efficiency. This shows that coaches familiar with an up-to-date knowledge can design a more effective development program with a larger likelihood of better performances by swimmers under them. Among those, the academic degree showed the largest contribution. The faster and more efficient swimmers were under coaches holding high academic degrees. Therefore, further and long-term learning over the coaching career, regardless of the path selected (higher academic degree and/or high-level coaching courses), concurrent to coaching experience is a must for young swimmers to deliver good performances. 


\section{DATA AVAILABILITY STATEMENT}

All datasets generated for this study are included in the article/supplementary material, further inquiries can be directed to the corresponding author.

\section{ETHICS STATEMENT}

The studies involving human participants were reviewed and approved by the University of Beira Interior Review Board. Written informed consent to participate in this study was provided by the participants' legal guardian/ next of kin.

\section{REFERENCES}

Barbosa, T. M., Bartolomeu, R., Morais, J. E., and Costa, M. J. (2019). Skilfull swimming in age-groups is determined by anthropometrics, biomechanics and energetics. Front. Physiol. 10:73. doi: 10.3389/fphys.2019. 00073

Barbosa, T. M., Bragada, J. A., Reis, V. M., Marinho, D. A., Carvalho, C., and Silva, A. J. (2010). Energetics and biomechanics as determining factors of swimming performance: updating the state of the art. J. Sci. Med. Sport 13, 262-269. doi: 10.1016/j.jsams.2009.01.003

Barbosa, T. M., Goh, W. X., Morais, J. E., Costa, M. J., and Pendergast, D. (2016). Comparison of classical kinematics, entropy, and fractal properties as measures of complexity of the motor system in swimming. Front. Psychol. 7:1566. doi: 10.3389/fpsyg.2016.01566

Barbosa, T. M., Morais, J. E., Marques, M. C., Silva, A. J., Marinho, D. A., and Kee, Y. H. (2015). Hydrodynamic profile of young swimmers: changes over a competitive season. Scand. J. Med. Sci. Sports 25, 184-196.

Barroso, R., Salgueiro, D. F., do Carmo, E., and Nakamura, F. Y. (2015). The effects of training volume and repetition distance on session rating of perceived exertion and internal load in swimmers. Int. J. Sports Physiol. Perform. 10, 848-852. doi: 10.1123/ijspp.2014-0410

Carter, J. G., Potter, A. W., and Brooks, K. A. (2014). Overtraining syndrome: causes, consequences, and methods for prevention. J. Sport Hum. Perform. $2,1-4$.

Cassidy, S. (2008). Olympic Swimming Training Too Hard on Young Athletes. Avaliable online at: https://www.independent.co.uk/sport/olympics/olympicswimming-training-too-hard-on-young-athletes-918054.html (accessed August 13, 2019).

Chatard, J. C., and Stewart, A. M. (2011). "Training load and performance in swimming," in World Book of Swimming: From Science to Performance, eds L. Seifert, D. Chollet, and I. Mujika (Hauppauge, NY: Nova Science Publishers), 359-373.

Cohen, J. (1988). Statistical Power Analysis for the Behavioral Sciences, 2nd Edn. Hillsdale, NJ: Lawrence Earlbaum Associates.

Costill, D. L., Kovaleski, J., Porter, D., Kirwan, J., Fielding, R., and King, D. (1985). Energy expenditure during front crawl swimming: predicting success in middle-distance events. Int. J. Sports Med. 6, 266-270. doi: 10.1055/s-20081025849

Craig, A. B., and Pendergast, D. R. (1979). Relationships of stroke rate, distance per stroke, and velocity in competitive swimming. Med. Sci. Sports. 11, $278-283$.

Crowcroft, S., Slattery, K., McCleave, E., and Coutts, A. J. (2020). Do athlete monitoring tools improve a coach's understanding of performance change? Int. J. Sports Physiol. Perform. [Epub ahead of print].

Denison, J. (2010). Planning, practice and performance: the discursive formation of coaches' knowledge. Sport Educ. Soc. 15, 461-478. doi: 10.1080/13573322. 2010.514740

Ferguson, C. J. (2009). An effect size primer: a guide for clinicians and researchers. Prof. Psychol. 40, 532-538. doi: 10.1037/a0015808

\section{AUTHOR CONTRIBUTIONS}

$\mathrm{DM}, \mathrm{TB}$, and JM conceived and designed the experiments, edited and reviewed. DM, PF, and JM performed the experiments. VL, $\mathrm{TB}$, and JM analyzed the data. DM, TB, VL, PF, AT, and JM wrote the original draft. All authors contributed to the article and approved the submitted version.

\section{FUNDING}

This work was supported by the National Funds (FCT Portuguese Foundation for Science and Technology) under the project UIDB/DTP/04045/2020.

Fry, M. D., and Gano-Overway, L. A. (2010). Exploring the contribution of the caring climate to the youth sport experience. J. Appl. Sport Psychol. 22, 294-304. doi: 10.1080/10413201003776352

Gilbert, W., and Côté, J. (2013). "Defining coaching effectiveness: focus on coaches' knowledge," in Routledge Handbook of Sports Coaching, eds P. Potrac, W. Gilbert, and J. Denison (Abingdon: Routledge International Handbooks), 147-159.

Grosso, M. R. (2006). Training theory: a primer on periodization. Coach. 33, 25-33. Kjendlie, P. L., and Stallman, R. K. (2008). Drag characteristics of competitive swimming children and adults. J. Appl. Biomech. 24, 35-42. doi: 10.1123/jab. 24.1.35

Kolmogorov, S. V., and Duplishcheva, O. A. (1992). Active drag, useful mechanical power output and hydrodynamic force coefficient in different swimming strokes at maximal velocity. J. Biomech. 25, 311-318. doi: 10.1016/00219290(92)90028-y

Lang, M., and Light, R. (2010). Interpreting and implementing the long term athlete development model: english swimming coaches' views on the (swimming) LTAD in practice. Int. J. Sports Sci. Coach. 5, 389-402. doi: 10. 1260/1747-9541.5.3.389

Lloyd, R. S., Cronin, J. B., Faigenbaum, A. D., Haff, G. G., Howard, R., Kraemer, W. J., et al. (2016). National strength and conditioning association position statement on long-term athletic development. J. Strength Cond. Res. 30, 14911509. doi: $10.1519 /$ jsc. 0000000000001387

Martinent, G., and Decret, J. C. (2015). Coping profiles of young athletes in their everyday life: a three-wave two-month study. Eur. J. Sport Sci. 15, 736-747. doi: 10.1080/17461391.2015.1051131

Monteiro, D., Marinho, D., Moutão, J., Vitorino, A., Antunes, R., and Cid, L. (2018). Reasons for dropout in swimmers, differences between gender and age and intentions to return to competition. J. Sports Med. Phys. Fitness. 58, 180-192.

Morais, J. E., Jesus, S., Lopes, V., Garrido, N., Silva, A., Marinho, D., et al. (2012). Linking selected kinematic, anthropometric and hydrodynamic variables to young swimmer performance. Pediatr. Exerc. Sci. 24, 649-664. doi: 10.1123/ pes.24.4.649

Morais, J. E., Silva, A. J., Marinho, D. A., Lopes, V. P., and Barbosa, T. M. (2017). Determinant factors of long-term performance development in young swimmers. Int. J. Sports Physiol. Perform. 12, 198-205. doi: 10.1123/ijspp.20150420

Morais, J. E., Silva, A. J., Marinho, D. A., Marques, M. C., Batalha, N., and Barbosa, T. M. (2016). Modelling the relationship between biomechanics and performance of young sprinting swimmers. Eur. J. Sport Sci. 16, 661-668. doi: 10.1080/17461391.2016.1149227

Nugent, F. J., Comyns, T. M., and Warrington, G. D. (2017). Quality versus quantity debate in swimming: perceptions and training practices of expert swimming coaches. J. Hum. Kinet. 57, 147-158. doi: 10.1515/hukin-2017-0056

Philippe, R. A., and Seiler, R. (2006). Closeness, co-orientation and complementarity in coach-athlete relationships: what male swimmers say about their male coaches. Psychol. Sport Exerc. 7, 159-171. doi: 10.1016/j.psychsport.2005.08.004 
Raudenbush, S. W., Bryk, A. S., Cheong, A. S., Fai, Y. F., Congdon, R. T., and du Toit, M. (2011). HLM 7: Hierarchical Linear and Nonlinear Modeling. Lincolnwood, IL: Scientific Software International.

Reade, I., Rodgers, W., and Hall, N. (2008). Knowledge transfer: how do high performance coaches access the knowledge of sport scientists? Int. J. Sports Sci. Coach. 3, 319-334. doi: 10.1260/174795408786238470

Rezania, D., and Gurney, R. (2014). Building successful student-athlete coach relationships: examining coaching practices and commitment to the coach. SpringerPlus. 3:383.

Salo, D., and Riewald, S. A. (2008). Complete Conditioning for Swimming. Champaign, IL: Human Kinetics.

Santi, G., Bruton, A., Pietrantoni, L., and Mellalieu, S. (2014). Sport commitment and participation in masters swimmers: the influence of coach and teammates. Eur. J. Sport Sci. 14, 852-860. doi: 10.1080/17461391.2014.91 5990

Valovich, M. T. C., Decoster, L. C., Loud, K. J., Micheli, L. J., Parker, J. T., Sandrey, M. A., et al. (2011). National athletic Trainers' association position statement: prevention of pediatric overuse injuries. J. Athl. Train. 46, 206-220. doi: 10.4085/1062-6050-46.2.206

Whitworth-Turner, C. M., Di Michele, R., Muir, I., Gregson, W., and Drust, B. (2019). Training load and schedule are important determinants of sleep behaviours in youth-soccer players. Eur. J. Sport Sci. 19, 576-584. doi: 10.1080/ 17461391.2018.1536171
Yustres, I., del Cerro, J. S., González-Mohíno, F., Peyrebrune, M., and GonzálezRavé, J. M. (2020). Analysis of world championship swimmers using a performance progression model. Front. Psychol. 10:3078. doi: 10.3389/fpsyg. 2019.03078

Zacca, R., Azevedo, R., Chainok, P., Vilas-Boas, J. P., Castro, F. A. S., Pyne, D. B., et al. (2020). Monitoring age-group swimmers over a training macrocycle: energetics, technique, and anthropometrics. J Strength Cond. Res. 34, 818-827. doi: $10.1519 /$ jsc. 0000000000002762

Zamparo, P., Cortesi, M., and Gatta, G. (2020). The energy cost of swimming and its determinants. Eur. J. Appl. Physiol. 120, 41-66. doi: 10.1007/s00421-01904270-y

Conflict of Interest: The authors declare that the research was conducted in the absence of any commercial or financial relationships that could be construed as a potential conflict of interest.

Copyright $\odot 2020$ Marinho, Barbosa, Lopes, Forte, Toubekis and Morais. This is an open-access article distributed under the terms of the Creative Commons Attribution License (CC BY). The use, distribution or reproduction in other forums is permitted, provided the original author(s) and the copyright owner(s) are credited and that the original publication in this journal is cited, in accordance with accepted academic practice. No use, distribution or reproduction is permitted which does not comply with these terms. 